\title{
Diagnóstico situacional de pessoas com deficiência acompanhadas em terapia ocupacional em uma unidade básica de saúde
}

\author{
Sabrina de Mello Rodrigues ${ }^{\mathrm{a}}$, Marta Aoki ${ }^{\mathrm{b}}$, Fátima Corrêa Oliver ${ }^{\mathrm{b}}$ \\ aPrograma de Residência Multiprofissional em Promoção da Saúde e Cuidado na Atenção Hospitalar, \\ Hospital Universitário, Universidade de São Paulo - USP, São Paulo, SP, Brasil. \\ ${ }^{b}$ Departamento de Fisioterapia, Fonoaudiologia e Terapia Ocupacional, Faculdade de Medicina, \\ Universidade de São Paulo - USP, São Paulo, SP, Brasil.
}

\begin{abstract}
Resumo: Trata-se de diagnóstico situacional de adultos com deficiência, identificados por uma Unidade Básica de Saúde, no município de São Paulo-SP. Buscou-se reconhecer as principais necessidades de saúde desses sujeitos para contextualizar, ampliar a atenção prestada e favorecer a ação interdisciplinar. A pesquisa foi realizada em duas fases: 1. Por meio de consultas aos prontuários e ao Sistema de Informações da Atenção Básica, para conhecimento de aspectos sociodemográficos e de saúde de 139 adultos; 2. Visitas domiciliares e entrevistas com 21 adultos com algum tipo de limitação, para realização do detalhamento do diagnóstico situacional. Os resultados mostram um número superior de sujeitos do sexo masculino, em idade economicamente ativa, porém excluídos do mundo do trabalho, com baixa escolaridade e poucas oportunidades de acesso a bens e serviços. Os locais de atendimento mais procurados foram a Unidade Básica de Saúde e o serviço hospitalar, com ênfase no recebimento de cuidados clínicos; porém, há identificação, por parte dos entrevistados, de necessidades no campo do tratamento em reabilitação. O estudo das condições de moradia, das dificuldades enfrentadas para a realização de deslocamentos, para o acesso a atividades de cultura e lazer, além da percepção e da vivência de situações de violência, apontou para fatores que se relacionam entre si e podem favorecer um ciclo de vulnerabilidade social e de não acesso a direitos sociais. É fundamental o reconhecimento de tais necessidades para se planejarem e implementarem ações contextualizadas pelas condições de vida dessa população, num esforço de compor uma rede de cuidados, integral e articulada.
\end{abstract}

Palavras-chave: Pessoas com Deficiência, Terapia Ocupacional, Atenção Primária à Saúde, Avaliação.

\section{Situational diagnosis of people with disabilities, receiving occupational therapy service in a basic health unit}

\begin{abstract}
This work is a situational diagnosis of adults with disabilities, identified by a basic health unit, in the city of São Paulo, SP. We sought to recognize their main health needs in order to contextualize, expand assistance and improve interdisciplinary action. The research was conducted in two phases. 1. through medical chart and Basic Assistance Information System review, for information of social-demographic as well as health aspects of 139 adults. 2. home visits and interviews with 21 adults with any type of limitation, aiming to detail the situational diagnosis. The results showed a higher number of male subjects, of working age, but excluded from the labor market, with low education and fewer opportunities of access to goods and services. The most sought care facilities were the Basic Health Unit and the hospital service, with emphasis on receiving clinical care. However, the interviewed identified needs in the rehabilitation treatment field. The study of living conditions, transport difficulties, access to culture and leisure activities, as well as perception and experiencing of violent situations, pointed to related factors that may facilitate some social vulnerability and impair access to social rights. The recognition of such needs is mandatory to plan and implement contextualized actions considering this population's critical life conditions in an effort to build a comprehensive and articulated care network.
\end{abstract}

Keywords: People with Disabilities, Occupational Therapy, Primary Health Care, Evaluation.

Autor para correspondência: Marta Aoki, Departamento de Fisioterapia, Fonoaudiologia e Terapia Ocupacional, Universidade de São Paulo, Rua Cipotânea, 51, CEP 05360-160, São Paulo, SP, Brasil, e-mail: aoki@usp.br

Recebido em Jun. 23, 2015; $1^{\text {a }}$ Revisão em Set. 4, 2015; Aceito em Out. 18, 2015. 


\section{Introdução}

No Brasil, a Atenção Primária em Saúde (APS) se configurou como principal porta de entrada dos usuários aos serviços de saúde, principalmente a partir do estabelecimento constitucional do Sistema Único de Saúde (SUS), o que inclui a criação de estratégias individuais e coletivas para a promoçáo e a proteção da saúde, a prevenção de agravos, o tratamento e a reabilitação do usuário. Apresenta, como atributos: o acesso, a continuidade do cuidado, a integralidade, a coordenação, a centralidade na família, a orientação para a comunidade e a adequação cultural na atençáo à saúde (STARFIELD, 2002).

Para aumentar a resolutividade da APS, foi proposta a Estratégia de Saúde da Família (ESF), que compreende a família como foco de sua assistência em saúde e prioriza a busca ativa de casos para intervençóes precoces, para facilitar o acesso dos usuários às Unidades Básicas de Saúde (UBS), aumentar a cobertura assistencial, proporcionar um acompanhamento longitudinal e integral das famílias, organizando atividades assistenciais baseadas no trabalho multiprofissional (CORBO; MOROSINI, 2005).

A atenção à saúde é um direito também das pessoas com deficiência (PCD), o que inclui o acesso à atençáo primária e a toda a rede assistencial do SUS. Neste estudo, consideram-se pessoas, com deficiência ou incapacidade, aquelas que têm deficiências física, mental, intelectual ou sensorial, de longa duraçáo, deficiências estas que, em interação com várias barreiras, podem impedir a sua participação completa e efetiva na sociedade em base de igualdade com outros (BRASIL, 2010).

Desde 1993, tem sido debatida a necessidade de criar condiçốes de acesso à atenção integral e equitativa a esse segmento da população. No entanto, o que se pode observar, são práticas prioritariamente realizadas nos setores secundários e terciários de assistência em saúde, em que a prática é baseada nos diferentes diagnósticos e patologias, com critérios de elegibilidade ligados ao perfil etário, à existência de apoio familiar e ao bom prognóstico de reabilitação (ROCHA; KRETZER, 2009). Esses critérios, muitas vezes, tornam a assistência inacessível às pessoas com quadros graves, rede familiar fragilizada, faixa etária mais elevada e situação econômica menos favorecida.

Nesse sentido, a APS tem um papel fundamental para possibilitar o acesso das $\mathrm{PCD}$ à atenção em saúde e, inclusive, a cuidados de reabilitação. A ESF, que conta com Agentes Comunitários em Saúde (ACS), pode viabilizar a identificação de necessidades e colaborar para a realização de diagnóstico situacional da comunidade, uma vez que estes profissionais são agentes facilitadores da comunicação entre o serviço de saúde e a população, o que, no caso das PCD deficiências, é essencial para construir possibilidades de acesso a direitos (MAIA et al., 2014).

O diagnóstico situacional tem, como objetivos, revelar, fotografar, mapear a situação que se deseja transformar e permitir a reflexão sobre a escolha das estratégias de intervençâo (MARTINS; BOGUS, 2004). Consiste em uma possibilidade de análise de informaçóes tanto de documentos como de dados sociodemográficos, relacionados, por exemplo, à atuação das Equipes de Saúde da Família, através do levantamento de dados relevantes (RIBEIRO et al., 2008), informaçôes estas que podem favorecer a proposição de práticas assistenciais mais contextualizadas com o perfil de necessidades das pessoas em determinado contexto territorial.

Parte das informaçóes relacionadas ao trabalho cotidiano das ESF está registrada no Sistema de Informações da Atenção Básica (SIAB), que é um instrumento frequentemente utilizado na realizaçáo do diagnóstico situacional das famílias, por caracterizar a situação sanitária, o perfil epidemiológico, a atençáo aos grupos de risco e o acompanhamento das açóes de saúde desenvolvidas no território (BITTAR et al., 2009). O ACS, em visitas domiciliares, necessita questionar as famílias sob sua responsabilidade sobre a presença de pessoas que apresentam diabetes, hipertensão arterial, hanseníase, uso de álcool e outras drogas, e também a presença de PCD no domicílio. Por ser um dado autorreferido pelo entrevistado, o que se observa é a subnotificação dos casos de deficiência cadastrados no $\mathrm{SIAB}$, quando comparados àqueles levantados pelo censo populacional, fato identificado, por exemplo, pelos responsáveis pelo planejamento da Rede de Cuidados à Pessoa com Deficiência no município de São Paulo, e indicado em seu documento orientador (SÃO PAULO, 2013). Essa subnotificação aponta para a condição de invisibilidade dessa população nos serviços de APS. Corrobora, também, a percepção dos familiares de que a deficiência deve ser abordada nos centros de reabilitação ou hospitais especializados e que, portanto, trabalhadores da atenção primária não estariam capacitados para lidar com esta população (SOUZA; ROCHA, 2010).

$\mathrm{Na}$ literatura internacional, não foram identificados estudos específicos sobre diagnóstico situacional relacionado às PCD e suas condições de participação social e restriçôes às atividades cotidianas. $\mathrm{O}$ que se observou foi a existência de estudos sobre a deficiência baseados na Classificação 
Internacional de Funcionalidade, Incapacidade e Saúde - CIF (ORGANIZAÇÃO..., 2003), que pretende auxiliar na compreensão das condiçôes de saúde, seus determinantes e efeitos, estabelecendo uma linguagem comum entre as naçôes que forneça base científica para investigações sobre estados de saúde. Madans et al. (2011) discutem sobre o desafio mundial de definir a deficiência e qualificar as informaçôes sobre essa população em uma linguagem universal e padronizada, baseada na Convenção da ONU sobre os direitos das PCD (RESENDE; VITAL, 2008) e na CIF, compreendendo que, para promover oportunidades de participação, são cruciais a identificação e a caracterização dessas pessoas.

Para Sampaio e Luz (2009), a CIF baseia-se na junção dos modelos médico e social, para compreender as várias dimensôes de saúde. A funcionalidade e a incapacidade humanas são concebidas como uma interação entre as condições de saúde e os fatores contextuais.

Foram identificados estudos sobre caracterizaçáo das pessoas com limitaçôes e suas necessidades a partir dos componentes da CIF: relação entre funcionalidade e fatores contextuais em sujeitos com sequelas de traumatismo cranioencefálico (TCE), com ênfase na complexidade das necessidades das pessoas acometidas por esse agravo, oferecendo importante subsídio para planejar e avaliar o processo de intervenção (LAXE et al., 2014); atividades e participação do ponto de vista de pacientes com doença pulmonar obstrutiva crônica, sendo observadas atividades relacionadas a complexas interaçôes sociais, relaçôes sociais informais, relaçóes familiares, conversar, manter uma posição do corpo, comer e preparar refeiçóes, o que pode promover uma avaliação mais centrada nos pacientes (MARQUES et al., 2013); influência dos fatores pessoais e ambientais em pacientes com sequelas de acidente vascular encefálico (BERZINA et al., 2013); caracterização da população com deficiência italiana, que recebe pensão por invalidez, com a descrição das condições de saúde, função e estruturas do corpo, atividades e participação, com maiores dificuldades encontradas na realização de atividades de vida diária, com forte impacto dos fatores ambientais (LEONARDI et al., 2009).

Contudo, prevalecem também estudos sociodemográficos de PCD, tendo como base dados censitários, como é o caso de países como Cuba, que identificou e caracterizou a população com deficiência intelectual (COBAS RUIZ et al., 2011) e PCD em geral (COBAS RUIZ et al., 2010), através de pesquisa ativa na comunidade, com cobertura de todo território nacional, cuja metodologia triplicou o número de PCD existente em pesquisa anterior. No Chile, realizou-se estudo sobre PCD física, maiores de 18 anos, moradores da região metropolitana e a relação entre qualidade de vida, tempo de aquisição da deficiência, saúde mental e fatores sociodemográficos (GONZÁLEZ et al., 2012). $\mathrm{Na}$ Bolívia, houve estudo sobre a ocorrência de diferentes tipos de deficiência e principais causas, com destaque para a aquisição de deficiência em acidentes (TABOADA-LUGO; MINAYA-RAMOS, 2012) e, no Peru, uma análise descritiva sobre as deficiências e incapacidades em crianças com atraso no desenvolvimento neuropsicomotor (CAMACHO-CONCHUCOS et al., 2012).

No Brasil, também tem havido esforços institucionais para caracterizar as PCD. O Censo de 2010 identificou cerca de $24 \%$ da população com pelo menos uma deficiência (INSTITUTO..., 2012). Contudo, existem poucos estudos que abordam as condiçóes de vida dessas pessoas, o que poderia subsidiar proposiçóes mais específicas para a garantia de acesso aos direitos sociais.

Nesta direção, Aoki et al. (2011) abordaram as condiçôes de vida de PCD a partir do levantamento em unidade básica de saúde e, dentre outras questóes, enfatizaram a fragilidade da rede de apoio social e a situaçáo de isolamento domiciliar da PCD e de seu cuidador; em outro estudo (AOKI; OLIVER, 2013), discutiram, a partir do discurso de PCD, as necessidades, como estudar, realizar tratamento em reabilitação, ter moradia digna, alimento, trabalho, benefício previdenciário, deslocar-se, passear e conversar, identificando aquelas necessidades relacionadas às condiçôes básicas de manutenção da vida e de acesso a direitos sociais. Othero e Ayres (2012) identificaram as necessidades de saúde das PCD pela ótica dos sujeitos, o que incluiu aspectos específicos de saúde e demais necessidades, que demandam uma atuação mais integral e com participação intersetorial; Souza e Pimentel (2012) abordaram as necessidades de saúde de PCD moradoras de bairro da cidade de Salvador e salientaram a condição multifacetada de exclusão e privação coletiva, traduzidas em necessidades de atendimentos especializados de saúde, melhorias da condiçáo de vida e o desejo de retorno a atividades que realizavam antes da aquisição da deficiência. Tais estudos apontam a atenção primária em saúde como possibilidade de atenção a essas pessoas.

Assim, considera-se fundamental ampliar o número de estudos específicos sobre diagnóstico situacional das PCD e suas condiçôes de participação social e restriçôes às atividades cotidianas junto a serviços de atenção primária em saúde, que subsidiem a criação, o monitoramento e a avaliação das intervençôes 
no campo das políticas públicas, que possam lidar com as necessidades apresentadas pelas PCD e suas famílias, com o objetivo de favorecer o acesso e o atendimento integral a saúde e reabilitação.

\section{Reabilitação com ênfase no território}

No Brasil, são escassos os registros de experiências de atenção comunitária a pessoas com limitaçóes em decorrência de deficiências e/ou risco de ruptura das redes sociais e de suporte. Tais experiências mostram-se pulverizadas no território nacional, sendo algumas ligadas a projetos de ensino e extensão de universidades, e poucas fomentadas por políticas públicas.

O presente trabalho é um estudo originado em uma Unidade Básica de Saúde (UBS), que conta com Estratégia de Saúde da Família (ESF) e equipe multidisciplinar, em parceria com curso de graduação em terapia ocupacional da Universidade de São Paulo, em que são desenvolvidas atividades de ensino, pesquisa e assistência às PCD moradoras da área de abrangência da UBS.

Dentre as atividades realizadas, destaca-se a implantação de grupos comunitários, como, por exemplo, o grupo de convivência "Família Mosaico". Criado em 2004, por terapeutas ocupacionais, com apoio das equipes de saúde, constituiu-se um grupo de convivência para pessoas com e sem deficiência; esse grupo, através da realização de artesanato utilizando a técnica do mosaico e de atividades políticas e culturais, tem como objetivo promover a participação social e a construção e/ou fortalecimento das redes de apoio social, e a defesa dos direitos das PCD (AOKI et al., 2014).

Os encontros ocorriam uma vez por semana, durante duas horas, sendo realizadas atividades de abordagem corporal, atividades artesanais em mosaico, discussôes coletivas sobre a organização do grupo, sobre temas referentes à condição da deficiência, aos direitos sociais e outros assuntos, que mobilizavam os participantes.

O trabalho desenvolvido no grupo facilitou a percepçáo de demandas apresentadas por parte das PCD identificadas por essa UBS, acumulando conhecimento e estratégias, que podem ser utilizadas para abordagem territorial em reabilitação.

Assim, considerando-se o contexto da APS e da atenção territorial em reabilitação, pretende-se apresentar estudo sobre diagnóstico situacional das condiçôes de vida de PCD. Essas informações podem ampliar o acesso, orientar e diversificar as práticas de saúde, no âmbito da APS e do trabalho em rede no território, para o cuidado da PCD e seus familiares.

\subsection{Procedimentos metodológicos}

Trata-se de um estudo ancorado em abordagem quantitativa, com caráter exploratório e descritivo das condiçóes de inserção social de adultos com limitaçôes nas atividades do cotidiano, por meio de um levantamento de dados junto ao serviço de atenção primária em saúde para caracterização sociodemográfica dessas pessoas e compreensão dessas limitaçôes. Foi desenvolvido entre janeiro e julho de 2011, e organizado em duas fases.

A primeira destas foi realizada a partir de consulta a um cadastro permanente de PCD identificadas pela terapia ocupacional e pelas equipes de ESF da UBS, num total de 340 pessoas cadastradas. Entre estas, havia $139(48,8 \%)$ com idades entre 18 e 60 anos, constituindo o grupo que participou deste estudo. A escolha por esta faixa etária ocorreu pela expressividade do número de adultos no cadastro, bem como sua situação de isolamento e pouca participaçáo social, quando este seria o grupo que, em teoria, poderia ter maior trânsito pelos espaços sociais.

O estudo caracterizou os 139 adultos com restriçōes na participaçáo social, que foram identificados - entre eles, os participantes do grupo "Família Mosaico" - por meio de levantamento de dados de prontuários e do cadastro do Sistema de Informaçôes da Atenção Básica -SIAB (BRASIL, 2014). No estudo dos prontuários, buscou-se conhecer o perfil sociodemográfico, o histórico de saúde-doença, moradia, trabalho e educação dos adultos identificados pelo serviço por meio de registro em roteiro pré-estabelecido. As informaçóes foram, então, inseridas e tratadas de forma descritiva com a utilização de planilhas do programa Microsoft Excel 2013.

No entanto, o preenchimento dessas informaçôes nos prontuários mostrou-se incompleto no que se referia à escolaridade (que não constava em $47 \%$ dos prontuários), à cor da pele (em $37,4 \%$ ), à profissão (em $36,6 \%)$ e à moradia (em 13,6\%), o que exigiu a utilização de outras estratégias de coleta de dados para sua complementação.

$\mathrm{Na}$ segunda fase do estudo, buscou-se o detalhamento do diagnóstico situacional, a partir de questionário com perguntas abertas e fechadas relacionadas a formas de viver, de trabalhar, de realizar atividades cotidianas, de compreender o processo saúde-doença-reabilitação e de acessar redes de serviços 
e de cuidados, grupos e benefícios sociais, sendo o questionário adaptado daquele utilizado durante estudos de necessidades de saúde junto ao Programa de Educação pelo Trabalho para a Saúde - PET Saúde da Família (CHIESA et al., 2012). Os questionários foram aplicados durante visitas domiciliares junto a 21 adultos com algum tipo de limitação, sem limites de idade, os quais, em algum momento, frequentaram o grupo de Convivência Família Mosaico desde sua criação, em 2004, até 2011.

O critério de inclusão foi a disponibilidade de receber os pesquisadores no domicílio, já que alguns participantes mudaram de endereço, outros faleceram e outros náo puderam participar, por motivo de agravo de sua condição de saúde. Dos 21 sujeitos que responderam ao questionário, 11 eram as próprias PCD. Os demais respondentes foram: mãe ou pai (5); filho ou filha (1), e irmão (4).

Foi realizada uma análise exploratória de dados, considerando-se a frequência percentual simples (PINHEIRO; TORRES, 2006) para os dados sociodemográficos (idade, sexo, cor da pele, estado civil, escolaridade, trabalho e renda, moradia) e para aqueles relacionados a problemas de saúde, como acesso ao cuidado, tipo de deficiência, tratamento e reabilitação, transporte, atividades de lazer, percepção de necessidades e violência ${ }^{1}$.

\section{Resultados e discussão}

\subsection{Caracterização dos participantes}

No estudo dos prontuários, foi observada a maior prevalência de pessoas do sexo masculino, sendo 74 homens (53,2\%) e 65 mulheres (46,7\%); em geral, tinham idade entre 18 e 29 anos (33,3\%), 40 e 49 anos (23\%) e 50 e 60 anos (23\%), caracterizando-se como uma população em idade economicamente ativa.

Quanto aos 21 entrevistados, observa-se que 14 eram homens e sete eram mulheres, com dez adultos na faixa etária entre 18 e 40 anos, e seis entre 41 e 60 anos. No grupo de convivência, participaram cinco pessoas com mais de 61 anos de idade, sendo todos do sexo masculino. Houve o mesmo número de sujeitos que se declararam brancos e pardos (dez pessoas em cada categoria), informaçôes sociodemográficas que se assemelham às obtidas no estudo dos prontuários.

Dados do SIAB indicam que adultos, na faixa etária entre 20 e 39 anos, são a maioria dos usuários, ou seja, o perfil da população moradora da área de abrangência da UBS é de jovens adultos em idade economicamente ativa. Tal fato é também observado entre os participantes do estudo dos prontuários, uma vez que houve maior prevalência de sujeitos com idades entre 18 e 29 anos.

Como demonstrado na apresentação dos dados, há um número superior de homens com incapacidades, aspecto que se diferencia dos estudos de caracterização da população que utiliza serviços de saúde, que, em sua maioria, refere as mulheres como maior percentual de usuários (KASSOUF, 2005).

Segundo Neri et al. (2003), no censo 2000, as mulheres eram a maioria entre PCD (54\%), dado influenciado pela maior longevidade feminina. Os homens eram maioria entre as pessoas com percepção de incapacidade (56\%), mais expostos à violência e acidentes, em particular durante a juventude. Esses dados foram confirmados no Censo 2010 (INSTITUTO..., 2012), que aponta as mulheres como maioria entre as PCD, com exceção daquelas com deficiências auditiva e intelectual. Também, na população geral cadastrada pela UBS estudada, há um número superior de mulheres, considerando todas as faixas etárias (BRASIL, 2014).

Nesse sentido, é fundamental a reflexão acerca do número superior de participantes do sexo masculino neste estudo. Teriam as mulheres com deficiência, moradoras da região da UBS, menos acesso à assistência? Ou ainda, seria possível considerar que as mulheres com incapacidades sofrem mais desvantagens sociais, com menores oportunidades de participação, no âmbito das iniciativas de educação, de trabalho remunerado e de participação comunitária, conforme discutem Nicolau, Schraiber e Ayres (2013).

Outro fator que pode colaborar para esse número reduzido de mulheres presentes no cadastro de PCD e participantes do estudo é o papel social, muitas vezes obrigatório, de cuidadora e dona de casa, que muitas mulheres com deficiência podem vir a exercer, o que pode comprometer a sua participaçáo social e sua circulação pela comunidade, afetando, concomitantemente, seu acesso aos serviços de saúde e reabilitação.

Estes aspectos mencionados podem apontar para a necessidade de instauração de outro olhar em relação à mulher com incapacidades, em que possa ser possível a incorporação de contextos assistenciais regidos por práticas de saúde ampliadas, considerando todo o conjunto de questóes que se situam nas necessidades de saúde de mulheres com incapacidades, captando aspectos psicossociais e socioculturais relacionados à questão das desigualdades existentes em relação ao gênero e à presença de deficiência (NICOLAU; SCHRAIBER; AYRES, 2013). Dados sobre o registro de cor referem que $36,6 \%$ eram brancos e 
$20,8 \%$, pardos. A populaçáo registrada como de cor negra era de apenas 5\%. A maior parte das pessoas nasceu no Estado de Sáo Paulo (44,4\%), seguido de Minas Gerais (5,7\%) ou alguns Estados da Região Nordeste, como a Bahia (6,4\%).

Em estudo sobre o censo, nos aspectos sociodemográficos, a etnia negra apresentava maior prevalência entre PCD, como também os maiores índices de incapacidades (NERI et al., 2003). Contudo, essa prevalência não se manifestou nessa UBS, podendo ser um indicativo de que haja menor acesso ao serviço de saúde e maior isolamento domiciliar daqueles de cor negra.

Quanto ao estado civil, 15 sujeitos entrevistados eram solteiros e seis apresentaram algum histórico de relação conjugal anterior ao acidente/doença que acarretou a incapacidade.

Considerando-se que o estudo é composto, principalmente, por jovens adultos, este dado pode ter grande significado, pois pode estar relacionado a vários aspectos que permeiam a deficiência, como, por exemplos, o isolamento domiciliar (AOKI et al., 2011; AOKI; OLIVER, 2013), a dependência nas atividades de vida diária, a falta de autonomia para gerir a vida afetiva e o preconceito, questôes estas que interferem diretamente no estabelecimento de um relacionamento conjugal.

As questôes afetivas são essenciais para que o indivíduo possa se perceber como alguém agradável e desejável. Em relação à $\mathrm{PCD}$, que, muitas vezes, possui um desvio da imagem socialmente aceita e difundida, é fundamental a criação de um espaço de diálogo e de mediação social, para que se possa desenvolver uma autoestima através do reforço das qualidades e potencialidades (MAIA, 2009).

\subsection{Escolaridade, trabalho e renda}

No estudo dos prontuários, 7,9\% das pessoas nunca haviam estudado, 23\% haviam completado o Ensino Fundamental e 17,3\%, o Ensino Médio. Aparentemente, a baixa escolaridade refletiu em suas situaçôes de trabalho: 13,6\% estavam desempregados; $8,6 \%$ realizavam trabalhos que exigiam baixa qualificação, e 14,3\% eram aposentados ou recebiam benefícios previdenciários. Também houve grande número de mulheres que exerciam especificamente a função de dona de casa (10\%). Nas entrevistas, observou-se um nível significativo de pessoas analfabetas, em número de nove.

Dos 21 entrevistadas, cinco eram aposentados por invalidez, sete recebiam o Benefício de Prestação Continuada (BPC) e dois estavam empregados e apenas um tinha trabalho formal. Quando foi questionado o motivo de essas pessoas não estarem inseridas no mercado de trabalho, nove pessoas afirmaram que a deficiência impedia a realizaçáo de trabalhos remunerados, afirmando serem incapazes de conseguir e manter um emprego. Uma pessoa afirmou que gostaria de trabalhar em casa, pois não conseguiria se locomover até o local de trabalho. A única pessoa que estava empregada no momento do estudo apresentava depressáo.

No passado, nove entrevistados já haviam trabalhado, sendo que a maioria destes desenvolveu trabalhos de limpeza e de manutenção, podendo ser um indicativo da relação entre baixa escolaridade e realização de trabalhos que envolvem esforço físico, em detrimento de trabalhos que demandam conhecimentos mais específicos, conforme também observado no primeiro núcleo do estudo.

Cavalcante et al. (2009), em estudo sobre a relação entre deficiência, violência e pobreza, e Souza e Carneiro (2007) discutem a forma como as desvantagens sociais, presentes na vida de muitas PCD, contribuem para uma situação de desvantagem no mercado de trabalho e consequente dificuldade de acessar direitos sociais. Estes fatores favorecem a manutenção de uma situação de falta de recursos financeiros, sendo esses aspectos considerados um ciclo de vulnerabilidade.

Garcia e Maia (2014) apresentaram estudo, baseado no censo demográfico de 2010, sobre inclusão no trabalho de PCD, considerando pessoas com níveis maiores de limitação física, sensorial ou cognitiva, e pessoas com limitaçóes funcionais mais leves. Segundo os autores, a taxa de participaçáo no mercado de trabalho das PCD (37\%) é inferior à taxa observada para as pessoas com limitaçóes mais leves $(58,1 \%)$ e as pessoas sem deficiência $(70,4 \%)$. Dentre as PCD e limitaçôes mais leves, a participação no mundo do trabalho é menor para PCD intelectual ou com grande dificuldade física. Além disso, a reserva de vagas no mercado de trabalho absorveria as $\mathrm{PCD}$ mais qualificadas, mantendo as de baixa qualificação em atividades vulneráveis, de baixa remuneração, e aumentando, dessa forma, a desigualdade entre os grupos de escolaridade.

Tais dados dialogam diretamente com o perfil observado na populaçáo do presente estudo, composta por jovens e adultos com baixa ou nenhuma escolaridade, apresentando incapacidades importantes, com destaque para as deficiências intelectuais e motoras mais severas.

Foi elevado o número de pessoas que recebiam benefícios previdenciários e sociais, mostrando a 
importância destes para a vida de PCD, pois, consistem, muitas vezes, na única fonte de renda de toda uma família (SANTOS, 2011). Em contrapartida, nas entrevistas, foi possível notar que algumas famílias descartavam a ideia da inserção das PCD no mercado de trabalho, para que não houvesse a suspensão do benefício (BPC), mesmo tendo conhecimento da alteração na legislação (BRASIL, 2011), que garante a retomada do recebimento do benefício, caso a PCD perca o emprego.

Portanto, o que se observa é que as políticas públicas focalizadas pouco contribuem para a participação da pessoa com deficiência no mundo do trabalho, considerando-se as fragilidades no acesso à educação formal e profissionalizante, e a reserva de vagas para PCD no mercado de trabalho. Para Souza e Carneiro (2007), a ação estatal mostra-se mais efetiva ao assegurar algum tipo de renda às $\mathrm{PCD}$ (aposentadoria, benefícios), o que tem garantido um acesso restrito a bens e serviços, mas insuficiente para assegurar o bem-estar das famílias, o que alimenta a relação entre pobreza e deficiência. Para os autores, as açóes focalizadas à população com deficiência devem ser conciliadas às açôes universais, que, de fato, promovam melhorias na oferta de serviços homogêneos, acessíveis e de qualidade ao conjunto da população.

\subsection{Saúde, acesso ao cuidado, deficiência e tratamento em reabilitação}

Os problemas de saúde que se destacaram no estudo dos prontuários foram a hipertensão arterial (29,4\%); a presença de dores $(25,1 \%)$; diabetes mellitus (15,8\%), e problemas ginecológicos (12,2\%). Também foram referidos: a obesidade $(7,5 \%)$; patologias cardíacas $(7,1 \%)$, e problemas na coluna vertebral (5,7\%). É importante ressaltar que 3,5\% dos sujeitos apresentavam contaminaçáo pelo vírus da HIV/AIDS.

Para o acompanhamento dos problemas de saúde, $79,8 \%$ das pessoas procuravam o serviço hospitalar e $76,9 \%$, a UBS. Em apenas $15,1 \%$ das moradias, havia pessoas cobertas por planos privados de saúde. Quanto aos serviços procurados na UBS, foram mais prevalentes: a consulta médica $(40,2 \%)$; a consulta com outros profissionais (21,5\%); a renovação de receita médica $(13,6 \%)$; a procura por laudos médicos $(12,9 \%)$; a consulta com profissionais da enfermagem (6,4\%), e a aferição de pressão arterial (5\%).

Quanto aos entrevistados, 33,3\% informaram ter hipertensão arterial sistêmica; destes, 23,8\% realizavam tratamento medicamentoso para a doença; 9,5\% possuíam comprometimentos respiratórios, e $38 \%$, outros problemas de saúde.

De modo geral, o estudo revelou a prevalência de sujeitos hipertensos e diabéticos, além de outras patologias, como as doenças cardíacas. Estes aspectos se relacionam e se afetam entre si, podendo ser indicativos da necessidade de adoçáo de modos de vida mais saudáveis, como o incentivo à realização de atividade física e à adesão a uma alimentação mais adequada. Esses aspectos indicam a importância do acompanhamento dessas pessoas pelo serviço, pois esses problemas de saúde podem ocasionar agravos das condiçôes de saúde e deficiência.

Um fator que interfere diretamente nessas alteraçóes sistêmicas é a alimentação e, nesse quesito do estudo, foi elevado o percentual de famílias que consumiam verduras, vegetais e frutas $(72,6 \%$ consumiam de uma a três vezes por semana), mas também foi recorrente o consumo de refrigerantes, doces e salgadinhos (57,2\%). Além disso, mostraram-se presentes comidas industrializadas, que contêm diversos conservantes e alto índice de açúcares e sódio.

Em estudo de Garcia (2004) sobre alimentação da população, foi discutida a representação social da alimentação, sendo constituída por valores culturais, econômicos e afetivos. Tornaram-se evidentes as dificuldades, encontradas por pessoas em dietas específicas, para seguir as prescriçóes quanto à alimentação adequada. Esse estudo teve metodologia e resultados semelhantes ao presente estudo, com a presença de alto consumo de comidas industrializadas.

Outro fator que interfere na alimentação e na saúde em geral é a saúde bucal: $66,7 \%$ dos sujeitos do estudo referiram problemas odontológicos, sendo que $47,6 \%$ realizavam acompanhamento com o dentista; observe-se que a maior parte desse atendimento foi realizada na UBS. Oito entrevistados apresentavam situação de hipodontia (ausência de seis dentes ou menos) e quatro de oligondotia (ausência de um número superior a seis dentes).

Diversos estudos demonstram que a falta de dentes afeta de forma considerável o desempenho das atividades diárias, pois desencadeia estímulos dolorosos ou alteraçôes psicológicas e emocionais que interferem no desempenho das atividades. Fatores, como idade avançada e situação econômica desfavorável, estáo intimamente relacionados à ausência de dentes, o que pode ser explicado pela dificuldade de acesso das pessoas em situação de pobreza aos serviços de saúde (MENDONÇA et al., 2010); dificuldades estas também são enfrentadas pelas $\mathrm{PCD}$, porém em 
maior grau de intensidade, quando estão presentes a situação de dependência e de pouca autonomia, e a condição de desvalorização social.

Observou-se, também, número significativo de pessoas infectadas pelo vírus HIV/AIDS (3,5\%), índice que foi superior ao número de pessoas com idade entre 15 e 49 anos infectadas em todo o Brasil (0,42\%), segundo aponta o boletim epidemiológico AIDS e DST (BRASIL, 2012). Esse alto índice pode ser resultado da falta de acesso de PCD às ações de prevenção e de informaçôes sobre doenças sexualmente transmissíveis.

Os entrevistados informaram que, na última vez que necessitaram de algum atendimento em saúde, procuraram a UBS. Quanto aos procedimentos mais procurados, foram citadas a consulta médica (62\%) e a consulta ao dentista (14,3\%). Assim, pode-se observar, como no levantamento dos dados de prontuários e do SIAB, a existência de um modelo hegemônico, com ênfase em ações clínicas e curativas, que compreende o processo saúde-doença, prioritariamente a partir do modelo biomédico (KASSOUF, 2005).

A procura da UBS por $76,9 \%$ dos entrevistados mostra que, para eles, este serviço é uma referência na assistência à saúde, no qual, inclusive, existe um histórico institucional de incentivo ao acesso de PCD, com discussões e ações para minimização das barreiras arquitetônicas e atitudinais presentes no serviço, o que tem promovido o acesso dessas pessoas ao serviço.

Porém, sabe-se que, no País, a realidade é bastante diferente. Estudos como os de França et al. (2010) e Castro et al. (2011) analisaram os problemas na acessibilidade aos serviços de saúde, principalmente de pessoas com mobilidade reduzida e deficiência visual. $\mathrm{O}$ que se percebe é um conflito existente entre a legislação que garante o direito de acesso às instituiçôes comunitárias e a organização dos serviços, que, muitas vezes, náo possibilita o atendimento da pessoa com algum tipo de incapacidade, o que contraria princípios fundamentais do SUS, como a universalização da assistência e a equidade.

Foi possível perceber que havia muitos prontuários com informaçôes incompletas, aspecto que pode comprometer o conhecimento das condiçóes de vida e saúde das pessoas na UBS, pois o registro também é uma das ferramentas que qualificam as ações individuais e coletivas das equipes de saúde. Um bom diagnóstico da comunidade pressupóe uma boa coleta de dados; dessa forma, o preenchimento inadequado de uma parcela considerável destes dados pode repercutir na não confiabilidade das informaçóes e no estabelecimento de práticas em saúde descontextualizadas (BRASIL, 2007).

Quanto à condição relacionada à presença de deficiência, observou-se, no estudo dos prontuários, maior prevalência de paralisia em partes do corpo $(31,6 \%)$; deformidade em alguma parte do corpo $(12,2 \%)$ e a dificuldade para deambular e se movimentar (31,6\%), e disfunçôes relacionadas ao Acidente Vascular Encefálico (AVE), patologia referida em $7,1 \%$ dos prontuários estudados. Ainda, $7,1 \%$ das pessoas tinham paralisia cerebral, $6,4 \%$ trauma cranioencefálico e $1,4 \%$ lesão medular. Observou-se também que 19,4\% dos sujeitos apresentavam dificuldades para falar, 8,6\% para enxergar, $8,6 \%$ para escutar e $5,7 \%$ apresentavam amputaçôes de membros.

Dentre as disfunçôes intelectuais e psicológicas, citaram-se: a dificuldade para compreender situaçóes $(15,8 \%)$; a perda de consciência ou convulsôes $(14,3 \%)$; as dificuldades em se relacionar com familiares (13,6\%); o comportamento agressivo $(12,2 \%)$; o estado frequente de ansiedade (9,3\%); o estado frequente de depressão (7,9\%), e o relato de situações fantasiosas/delírios (4,3\%). Dentre as pessoas que apresentavam sofrimento psíquico, apenas 9,3\% realizavam acompanhamento em serviços especializados em saúde mental.

Dos 21 entrevistados, 12 (57,14\%) apresentavam a deficiência desde o nascimento e sete $(33,3 \%)$ tinham adquirido a deficiência havia mais de cinco anos. Dentre os diagnósticos mais frequentes, podem ser citadas a deficiência intelectual, o acidente vascular encefálico, a lesão medular, a paralisia cerebral e a Síndrome de Down. Duas pessoas apresentavam sofrimento psíquico.

Metade dos entrevistados $(52,4 \%)$ apresentava dificuldade para movimentar-se, com destaque aos sujeitos que tinham dificuldades para se deslocar da cama para a cadeira (38\%) e aqueles com dificuldades para subir e descer escadas (62\%). Dentre os motivos relatados para dificuldades apresentadas para locomoçáo, 52,3\% referiram problemas de saúde; $38 \%$, a falta de um cuidador disponível e $47,6 \%$, a presença de barreiras arquitetônicas, que dificultam os acessos e a circulação. Observou-se também que $47,6 \%$ necessitavam do auxílio de alguém para superar dificuldades de locomoção, $38,1 \%$ utilizavam cadeira de rodas e, ainda, 9,5\% se valiam de equipamentos como muletas e órteses de posicionamento para membros inferiores.

A habilidade de comunicação e a capacidade de aprender a ler e escrever foram percebidas como uma importante dificuldade em 15 dos entrevistados 
$(71,4 \%)$. Cinco pessoas $(23,8 \%)$ relataram perda de memória; quatro (19\%), delírios; dez (47,6\%), perda de consciência ou convulsão contínua ou esporadicamente, e 11 (52,4\%), excesso de raiva, tristeza, alegria e ansiedade. Nenhum dos entrevistados relatou uso problemático de álcool, drogas ou medicamentos. Cinco sujeitos $(23,8 \%)$ utilizavam antidepressivos e quatro (19\%) realizavam tratamento em saúde mental, na UBS e no Centro de Atenção Psicossocial (CAPS).

Os dados do último Censo (INSTITUTO..., 2012) auxiliam na discussão sobre a magnitude e a relevância dos problemas da populaçáo com deficiência no País. O estudo revelou que $23,9 \%$ da população brasileira apresentavam algum tipo de deficiência, sendo a deficiência mais mencionada a deficiência visual, seguida pelas deficiências motora, auditiva e intelectual.

Contudo, o diagnóstico situacional realizado apresenta uma frequência maior de deficiências motoras e intelectuais. Esta diferença entre os estudos pode ser resultado da forma escolhida para coleta dos dados. O Censo considerou pessoas com diversos níveis de dificuldade, como, por exemplo, dificuldade para enxergar sem auxílio de óculos. No presente estudo, foram considerados como deficiências os déficits sensoriomotores, que acarretam importantes limitaçôes para realização de atividades de vida diária, locomoção, aprendizagem e participação social.

As disfunçôes mentais e psicológicas foram significantemente mencionadas nos prontuários, mas, apesar dessas indicaçóes, apenas 9,3\% realizavam tratamento especializado. Para Bandeira et al. (2003), as habilidades de vida cotidiana possibilitam o desenvolvimento do sentimento de competência, domínio ou autonomia nas atividades de vida diária. Desta maneira, a assistência realizada no âmbito territorial pode ser avaliada como mais eficaz, por considerarem o cotidiano do indivíduo e o território que o permeia. Assim, é fundamental a identificação pela ESF dos casos de sofrimento psíquico presentes no território, para adoção de uma atenção e intervenção contextualizadas, levando em conta, também, a rede de serviços de apoio psicossocial da regiáo.

Apenas seis pessoas $(28,6 \%)$ realizavam algum tipo de tratamento em reabilitação. Destes, cinco referiram o tratamento em terapia ocupacional, reflexo do trabalho desenvolvido no grupo de convivência "Família Mosaico".

O fato de este grupo de convivência ter sido mencionado como único tratamento atual realizado por essas pessoas permite a reflexão sobre a importância do mesmo, não somente como instrumento para aumentar a participação social dessas pessoas, mas também como um espaço em que se possa realizar reabilitação e que favoreça o acesso ao serviço de saúde. Esse tipo de iniciativa pode ser considerado como abordagem a ser utilizada na APS, que facilita a identificação das PCD, a caracterização de suas principais demandas e a circulação pelo território e pelos espaços comunitários.

Quanto à necessidade de outro tipo de tratamento, 15 entrevistados (71,5\%) afirmaram apresentar esta demanda, sendo que o tratamento de Fisioterapia e Fonoaudiologia foram os mais citados. Também foram informados, como necessidades atuais, o atendimento em psicologia, a hidroterapia, a neurologia e a equoterapia, o que demonstra reconhecimento, por parte das famílias, da existência de amplo leque de alternativas assistenciais, atendimentos presentes em serviços como centros de reabilitação; entretanto, muitas vezes, estes se mostram inacessíveis aos usuários pela existência de critérios de elegibilidade, que excluem pessoas com idade elevada e sem prognóstico de reabilitação.

\subsection{Moradia}

O conhecimento das condiçôes de habitação da população é fundamental para o estabelecimento de açôes que visem à promoçáo da qualidade de vida dos indivíduos, das famílias e da comunidade. $\mathrm{Na}$ ESF, procura-se ultrapassar o conceito de saúde focalizado apenas no indivíduo e busca-se contextualizar a saúde no espaço físico, social e relacional. Segundo Azeredo et al. (2007, p. 744), é fundamental considerar que:

A habitação é um agente de saúde de seus moradores e relaciona-se com o território geográfico e social onde se assenta; os materiais usados para sua construção, a segurança e qualidade dos elementos combinados, o processo construtivo, a composição espacial, a qualidade dos acabamentos, o contexto global do entorno (comunicaçóes, energia, vizinhança) e a educação em saúde e ambiente de seus moradores sobre estilos e condiçóes de vida saudável.

Quanto às condiçôes de moradia, os dados de prontuário demonstraram que a maioria dos sujeitos habitava casas ou apartamentos (82\%) e 4,3\% moravam em habitaçôes construídas em terrenos irregulares, sendo a mesma porcentagem observada nos entrevistados. As famílias eram compostas por duas $(13,6 \%)$, três $(19,4 \%)$, quatro $(20,1 \%)$ ou cinco $(16,5 \%)$ pessoas. 
Em relação ao número de cômodos existentes nas residências, houve variação de dois a oito cômodos, sendo mais prevalentes os domicílios com cinco cômodos (39,5\%), três cômodos $(17,9 \%)$ e seis cômodos (12,9\%). Observou-se, em alguns casos, número elevado de moradores por domicílio em relaçáo também ao número de cômodos nas residências, o que pode indicar condiçôes mais precárias de habitação.

Durante as entrevistas, foi possível observar as barreiras arquitetônicas presentes nas moradias. Dos 21 domicílios, $62 \%$ possuíam escadas e degraus na entrada, e 42,8\% tinham degraus ou escada entre os cômodos. Onze moradias apresentavam móveis ou objetos que dificultavam a circulação, pisos escorregadios, falhas ou irregularidades nos mesmos. No caso das residências habitadas por pessoas com dificuldades para enxergar, oito possuíam obstáculos para circulação. Estes são aspectos que podem acarretar acidentes domésticos ou isolamento em determinados cômodos da residência.

\subsection{Transporte e atividades de lazer}

Quanto ao uso de transporte, 62\% dos entrevistados utilizavam ônibus comum, 28,7\%, ônibus adaptado, e $38 \%$, o serviço de transporte para pessoas com mobilidade reduzida (ATENDE). Porém, a maioria (62\%) também utilizava carro particular para realização de deslocamentos. As dificuldades para utilização do transporte coletivo foram presença de barreira "arquitetônica" e falta de um cuidador, com $38 \%$, e o comprometimento de saúde, citado por $28 \%$ dos entrevistados.

Das pessoas que participaram do estudo, $85,7 \%$ informaram seguir algum tipo de religião, sendo a maioria evangélica $(42,8 \%)$ ou católica $(33,3 \%)$. A frequência de ida à igreja/culto variava, podendo ser semanalmente $(38,1 \%)$, esporadicamente $(19 \%)$ ou mais de uma vez por semana $(14,3 \%)$.

Quanto às relaçôes pessoais, $57,2 \%$ dos sujeitos alegaram contato com outras pessoas de sua faixa etária; $85,7 \%$ mantinham contato com vizinhos e pessoas que residem em seu domicílio, e 71,4\%, com parentes que moram em outras residências. No aspecto da participação, 14,3\% dos sujeitos participavam de movimentos e organizações políticas, sendo citados os movimentos sociais e o Conselho Municipal das Pessoas com Deficiência. Em situaçôes em que é necessário o auxílio socioeconômico, 42,9\% das pessoas recorriam a alguém, podendo ser familiares, amigos e vizinhos.
Dentre as atividades de lazer realizadas pelos entrevistados, destacaram-se aquelas realizadas na própria moradia (ouvir música, assistir à televisão) e que não envolvem deslocamentos, podendo isso ser reflexo das dificuldades mencionadas para locomoçấo e utilização do transporte público, da falta de acessibilidade dos espaços públicos e privados, e da existência de poucos espaços culturais e de lazer nesse bairro periférico. Ainda foi possível observar que os jovens e adultos, em sua maioria, não realizavam atividades características de sua faixa etária.

Em estudo sobre as necessidades das PCD, observou-se que as situaçóes de lazer e passeio são desejadas, porém o que tem prevalecido é a condição de isolamento social, em que são raras as oportunidades de participação comunitária (AOKI; OLIVER, 2013).

Para D'Antino e Mazzota (2011), atividades de cultura, educação e lazer são cruciais para o desenvolvimento sociocultural, favorecendo a construção de valores e atitudes que podem consolidar espaços estruturados com poder de mediação para inclusão social da pessoa com deficiência, assim como para qualquer outro sujeito.

\subsection{Percepção de demandas e necessidades/violência}

Das pessoas que responderam ao questionário, $90,5 \%$ afirmaram ter dificuldades para as quais necessitam de ajuda, sendo as mais mencionadas os problemas financeiros, a falta de atendimento de qualidade em saúde e a falta de apoio dos familiares para o cuidado das PCD. Para sanar essas dificuldades, os entrevistados relataram a necessidade de um aumento na renda das famílias, sendo através de empregos melhor remunerados ou de benefícios sociais. A necessidade de tratamento especializado para as PCD também foi mencionada.

Quanto a experiências de violência, os entrevistados foram questionados se já haviam sido expostos ou presenciado alguma situação de violência, e 47,6\% responderam afirmativamente. Foram referidos, na entrevista: a violência física ou psicológica $(47,6 \%)$; a falta de cuidados cotidianos (5\%); o isolamento $(4,8 \%)$, e a obrigatoriedade de cuidar de familiares ou realizar afazeres domésticos $(4,8 \%)$.

Para Cavalcante et al. (2009), a pobreza também está relacionada com as situaçóes de violência e violação dos direitos. A mesma autora afirma que a violência é maior quando há uma combinação entre fatores de risco (o isolamento social, o estigma e o estresse psicossocial); fatores de proteção (cuidados 
pessoais e sociais, acesso a serviços, escola e benefícios), e os de vulnerabilidades associadas à deficiência (impossibilidade de andar, enxergar, ouvir, de pedir ajuda, de compreensáo e discernimento de situações, que envolvem noções de certo e errado).

Esses fatores evidenciam tanto a dificuldade em identificar as situaçôes de violência vivenciadas pelas PCD e suas famílias como para lidar com as situaçôes objetivas de violência, sendo necessária uma atenção interdisciplinar que considere a complexidade dos aspectos envolvidos e a promoçáo de açốes no combate aos fatores de risco e o incentivo das formas de proteção e promoção dos direitos das PCD e sua família.

Assim, é fundamental que os profissionais da UBS estejam atentos para possíveis casos de violência, expressos por situaçóes que incluem desde a negligência de cuidados até agressôes verbais, físicas, restrição ao direito de ir e vir, e de acessar recursos financeiros relacionados aos benefícios sociais. A atenção dos profissionais de saúde a esses aspectos é fundamental, mesmo que ainda haja desconhecimento do que seriam consideradas as situaçóes de violência e que ainda exista certo receio de realizar a notificação dessas situaçóes, tanto por parte da equipe como de familiares.

\section{Considerações finais}

O estudo possibilitou melhor compreensão sobre aspectos relacionados ao ciclo de vulnerabilidade associado à deficiência, incluindo elementos como incapacidades, falta de recursos financeiros, baixa escolaridade, trabalhos mal remunerados, violência e questốes de gênero, que se afetam mutuamente e demandam ações interdisciplinares e intersetoriais.

No que diz respeito à relação entre pobreza e deficiência, é urgente o estabelecimento de políticas públicas sociais que sejam universais, regulares e de qualidade, e que não se resumam apenas a apoios financeiros que perpetuam a condição da pessoa com deficiência e sua família à subsistência, com restrição ao acesso a bens e serviços essenciais para manutenção da qualidade de vida.

A invisibilidade da população com deficiência nos serviços de atenção primária a saúde deve ser questionada e mecanismos de promoção de acesso podem ser pensados e acionados pelas equipes, considerando-se a importância dos serviços territoriais e comunitários de saúde como porta de entrada da rede de cuidado de toda a população. Dessa forma, pode-se contribuir para a desconstruçáo do imaginário de que as açôes em saúde e reabilitação a essa população deveriam estar restritas aos serviços especializados em reabilitação, que, muitas vezes, são inacessíveis para as PCD e suas famílias, pelos motivos expostos.

O diagnóstico situacional apresentado, relacionado à condiçáo de vida e às necessidades de adultos com deficiências, moradores de região periférica da cidade de São Paulo, pode ampliar o diálogo com o serviço de saúde, para o desenvolvimento de práticas - tanto de promoção da saúde, prevençáo de doenças, como de diagnóstico, tratamento e reabilitação - mais contextualizadas pelas condiçôes de vida indicadas pelo perfil sociodemográfico, pelo perfil de saúde das pessoas e pelas dificuldades de acesso a direitos que puderam ser identificados. Como exemplo de intervenção prática, pode-se citar a criação de grupos de convivência para pessoas com e sem deficiência na comunidade, que agregaram sujeitos para realização de atividades significativas de lazer e cultura, e discussão sobre a condiçãa de vida, os direitos e as formas de acessá-los.

Urge o estabelecimento de uma atuação intersetorial para lidar com questóes tão complexas como as apresentadas neste estudo. Nesse sentido, o serviço de atenção territorial em saúde tem um papel de destaque como interlocutor das discussóes com os demais serviços do território, que possam, de forma institucional, articulada e pautada em políticas públicas, construir propostas que lidem efetivamente com as necessidades apresentadas pelas PCD e suas famílias. Necessidades estas que se mostram nos campos da educação, do trabalho, da moradia, do transporte, do lazer, da cultura e da proteção social.

A discussão trazida pelo estudo promove a necessidade de aprofundamento da formação de profissionais de terapia ocupacional, em particular, e de saúde, de maneira geral, com o objetivo de melhor prepará-los para reconhecer necessidades e demandas das PCD, e, dessa maneira, contextualizar a atenção realizada e os desafios colocados para a implementação do exercício do direito à saúde e à reabilitação para esse segmento da populaçáo.

\section{Agradecimentos}

A Bruna Arruda e Felipe Gemelgo, que contribuíram para a coleta de dados durante o Programa Aprender com Cultura e Extensão da USP.

\section{Referências}

AOKI, M. et al. Grupo de Convivência Família Mosaico: participaçáo de pessoas com deficiência na comunidade. O Mundo da Saúde, São Paulo, v. 38, n. 2, p. 149- 
158, 2014. Disponível em: <http://www.saocamilo-sp. br/pdf/mundo_saude/155562/A03.pdf>. Acesso em: 20 set. 2014.

AOKI, M.; OLIVER, F. C. Pessoas com deficiência moradoras de bairro periférico da cidade de São Paulo: estudo de suas necessidades. Cadernos de Terapia Ocupacional da UFSCar, São Carlos, v. 21, n. 2, p. 391-398, 2013. http://dx.doi.org/10.4322/cto.2013.040.

AOKI, M.; OLIVER, F. C.; NICOLAU, S. M. Consideraçóes acerca das condiçóes de vida das pessoas com deficiência a partir de um levantamento em uma unidade básica de saúde de um bairro periférico do município de São Paulo. O Mundo da Saúde, São Paulo, v. 35, n. 2, p. 169-178, 2011. Disponível em: <http://saocamilo-sp. br/pdf/mundo_saude/84/169-178.pdf>. Acesso em: 15 fev. 2012.

AZEREDO, C. M. et al. Avaliação das condições de habitação e saneamento: a importância da visita domiciliar no contexto do Programa de Saúde da Família. Ciência \& Saúde Coletiva, Rio de Janeiro, v. 12, n. 3, p. 743-753, 2007.

BANDEIRA, M.; LIMA, L. A.; GONÇALVES, S. Qualidades psicométricas no papel da Escala de Habilidades de Vida Independente de pacientes psiquiátricos (ILSS-BR). Archives of Clinical Psychiatry, São Paulo, v. 30, n. 4, p. 121-125, 2003. http://dx.doi.org/10.1590/ S0101-60832003000400002.

BERZINA, G. et al. Exploration of some personal factors with the International Classification of Functioning, Disability and Health core sets for stroke. Journal of Rehabilitation Medicine, Uppsala, v. 45, n. 7, p. 609615, 2013. http://dx.doi.org/10.2340/16501977-1171. PMid:23757183.

BITTAR, T. O. et al. O Sistema de Informação da Atenção Básica como ferramenta da gestão em saúde. Revista da Faculdade de Odontologia, Passo Fundo, v. 14, n. 1, p. 77-81, 2009. Disponível em: <http://www.upf.br/ seer/index.php/rfo/article/view/675/434>. Acesso em: 28 jan. 2015.

BRASIL. Ministério da Saúde. Conselho Nacional de Secretários de Saúde - CONASS. Atenção primária e promoção da saúde. Brasília, 2007. Disponível em: <http://bvsms.saude.gov.br/bvs/publicacoes/colec_progestores_livro8.pdf>. Acesso em: 15 set. 2013.

BRASIL. Presidência da República. Secretaria dos Direitos Humanos. Secretaria Nacional de Promoção dos Direitos das Pessoas com Deficiência. Convençâo sobre os Direitos das Pessoas com Deficiência. Brasília, 2010.

BRASIL. Lei no 12.435 de 06 de julho de 2011. Altera a Lei n. 8.742, de 7 de dezembro de 1993, que dispóe sobre a organização da Assistência Social. Dispóe sobre a organização do setor e institui o Sistema Único de Assistência Social (SUAS). Diário Oficial [da] República Federativa do Brasil, Poder Executivo, Brasília, DF, 7 jul. 2011.
BRASIL. Ministério da Saúde. Boletim epidemiológico AIDS e DST. Brasília, 2012. Disponível em: <http:// www.aids.gov.br/sites/default/files/anexos/publicacao/2012/52654/boletim_jornalistas_pdf_22172.pdf>. Acesso em: 13 ago. 2013.

BRASIL. Ministério da Saúde. Sistema de Informação da Atenção Básica. Brasília, 2014.

CAMACHO-CONCHUCOS, H. T.; FAJARDO-CAMPOS, P.; ZAVALETA DE FLORES, E. Análisis descriptivo sobre deficiencias y discapacidades del desarrollo psicomotor en pacientes atendidos en el Instituto Nacional de Rehabilitación: 2006-2008. Anales de la Facultad de Medicina, Lima, v. 73, n. 2, p. 119-126, 2012. Disponível em: <http://revistasinvestigacion.unmsm.edu.pe/index.php/anales/article/ view/829/659>. Acesso em: 20 jan. 2015.

CASTRO, S. S. et al. Acessibilidade aos serviços de saúde por pessoas com deficiência. Anales de la Facultad de Medicina, Lima, v. 45, n. 1, p. 99-105, 2011. http:// dx.doi.org/10.1590/s0034-89102010005000048.

CAVALCANTE, F. G. et al. Diagnóstico situacional da violência contra crianças e adolescentes com deficiência em três instituições do Rio de Janeiro. Ciência \& Saúde Coletiva, Rio de Janeiro, v. 14, n. 1, p. 45-56, 2009. http:// dx.doi.org/10.1590/S1413-81232009000100010.

CHIESA, A. M. et al. PET Saúde da Família: estudo para captação de necessidades de saúde. São Paulo: [s.n.], 2012. Relatório de pesquisa apresentado como parte das atividades realizadas no âmbito do PET Saúde da Família: período de 2009 a 2012. Relatório preliminar.

COBAS RUÍZ, M. et al. Caracterización epidemiológica de las personas con discapacidad en Cuba. Revista Cubana de Salud Pública, La Habana, v. 36, n. 4, p. 306-310, 2010. http://dx.doi.org/10.1590/S086434662010000400004 .

COBAS RUIZ, M. et al. Caracterización epidemiológica y social de las personas con discapacidad intelectual en Cuba. Revista Cubana de Salud Pública, Cuba, v. 37, n. 1, p. 34-43, 2011. http://dx.doi.org/10.1590/S086434662011000100005 .

CORBO, A. A.; MOROSINI, M. V. G. C. Saúde da família: história recente da reorganização da atenção à saúde. In: ESCOLA POLITÉCNICA DE SAÚDE JOAQUIM VENÂNCIO (Org.). Textos de apoio em politicas de saúde. Rio de Janeiro: Fiocruz, 2005. p. 157-181.

D’ANTINO, M. E. F.; MAZZOTA, M. J. S. Inclusão social de pessoas com deficiências e necessidades especiais: cultura, educação e lazer. Saúde e Sociedade, São Paulo, v. 20, n. 2, p. 377-389, 2011. http://dx.doi. org/10.1590/S0104-12902011000200010.

FRANÇA, I. S. X. et al. Violência simbólica no acesso das pessoas com deficiência às Unidades Básicas de Saúde. Revista Brasileira de Enfermagem, Brasília, v. 63, n. 6, p. 944-970, 2010.

GARCIA, R. W. D. Representaçóes sobre o consumo alimentar e suas implicaçóes em inquéritos alimentares: 
estudo qualitativo em sujeitos submetidos a prescrição dietética. Revista de Nutrição, Campinas, v. 17, n. 1, p. 15-28, 2004. http://dx.doi.org/10.1590/S141552732004000100002 .

GARCIA, V. G.; MAIA, A. Características da participação das pessoas com deficiência e/ou limitação funcional no mercado de trabalho. Revista Brasileira de Estudos de População, São Paulo, v. 31, n. 2, p. 395-418, 2014. http://dx.doi.org/10.1590/S010230982014000200008 .

GONZÁLEZ, S. et al. Calidad de vida en pacientes con discapacidad motora según factores sociodemográficos y salud mental. Revista Chilena de Neuro-psiquiatría, Santiago, v. 50, n. 1, p. 23-34, 2012. http://dx.doi. org/10.4067/S0717-92272012000100003.

INSTITUTO BRASILEIRO DE GEOGRAFIA E ESTATÍSTICA - IBGE. Censo Demográfico 2010. Brasília, 2012. Disponível em: <http://censo2010.ibge.gov.br/ resultados>. Acesso em: 3 mar. 2013.

KASSOUF, A. L. Acesso aos serviços de saúde nas áreas urbanas e rural do Brasil. Revista de Economia e Sociologia Rural, Brasília, v. 43, n. 1, p. 29-44, 2005. http://dx.doi. org/10.1590/S0103-20032005000100002.

LAXE, S. et al. Profiling of patients with traumatic brain injury: an international professional survey. Disability and Rehabilitation, London, v. 36, n. 1, p. 82-88, 2014. http://dx.doi.org/10.3109/09638288.2013.780641. PMid:23596999.

LEONARDI, M. et al. The White Book on Disability in Italy: an ICF-based Italian survey. Disability and Rehabilitation, London, v. 31, n. SE1, p. 40-45, 2009. http://dx.doi.org/10.3109/09638280903317740. PMid:19968533.

MADANS, J. H.; LOEB, M. E.; ALTMAN, B. M. Measuring disability and monitoring the UN convention on the rights of persons with disabilities: the work of the Washington group on disability statistics. Public Health, Hyattsville, v. 11, n. SE 4, p. 2-8, 2011. PMid:21624190.

MAIA, A. C. B. A importância das relações familiares para sexualidade e autoestima de pessoas com deficiência física. Portal dos Psicólogos, Porto, v. 1, p. 1-10, 2009. Disponível em: <http://www.psicologia.pt/artigos/textos/A0515.pdf>. Acesso em: 27 jan. 2015.

MAIA, E. V.; PAGLIUCA, L. M. F.; ALMEIDA, P. C. Aprendizagem do agente comunitário de saúde para identificar e cadastrar pessoas com deficiência. Acta Paulista de Enfermagem, São Paulo, v. 27, n. 4, p. 326-332, 2014. http://dx.doi.org/10.1590/1982-0194201400055.

MARQUES, A. et al. Comprehensive ICF core set for obstructive pulmonary diseases: validation of the activities and participation component through the patient's perspectiv. Disability and Rehabilitation, London, v. 35, n. 20, p. 1686-1691, 2013. http://dx.doi.org/10.3109/0 9638288.2012.750691. PMid:23343360.
MARTINS, M. C. E. F. N.; BOGUS, C. M. Consideraçóes sobre a metodologia qualitativa como recurso para o estudo das açóes de humanização em saúde. Saúde $e$ Sociedade, São Paulo, v. 13, n. 3, p. 44-57, 2004. Disponível em: <http://www.revistas.usp.br/sausoc/article/ view/7125/8599>. Acesso em: 28 jan. 2015

MENDONÇA, B. M. C. et al. Impacto do número de dentes presentes do desempenho de atividades diárias: estudo piloto. Ciencia \& Saúde Coletiva, Rio de Janeiro, v. 15, n. 3, p. 775-784, 2010. http://dx.doi.org/10.1590/ S1413-81232010000300020.

NERI, M. et al. Retratos da deficiência no Brasil. Rio de Janeiro: Fundação Banco do Brasil, FGV/CPS, 2003.

NICOLAU, S. M.; SCHRAIBER, L. B.; AYRES, J. R. C. M. Mulheres com deficiência e sua dupla vulnerabilidade: contribuiçóes para a construção da integralidade em saúde. Ciência \& Saúde Coletiva, Rio de Janeiro, v. 18, n. 3, p. 863-872, 2013. http://dx.doi.org/10.1590/ S1413-81232013000300032.

ORGANIZAÇÃO MUNDIAL DA SAÚDE - OMS. Organização Pan-Americana da Saúde - OPAS. Classificação Internacional de Funcionalidade, Incapacidade e Saúde: CIF. São Paulo: EDUSP, 2003.

OTHERO, M. B.; AYRES, J. R. C. M. Necessidades de saúde da pessoa com deficiência: a perspectiva dos sujeitos por meio de histórias de vida. Interface Comunicação, Saúde, Educação, Botucatu, v. 16, n. 40, p. 219-233, 2012. http://dx.doi.org/10.1590/S141432832012005000010 .

PINHEIRO, R. S.; TORRES, T. Z. G. Análise exploratória de dados. In: MEDRONHO, R. A. et al. (Eds.). Epidemiologia. São Paulo: Atheneu, 2006. p. 227-244.

RESENDE, A. P. C.; VITAL, F. M. P. V. A convenção sobre os direitos das pessoas com deficiência. Brasília: Secretaria Especial dos Direitos Humanos, 2008.

RIBEIRO, L. C. C. et al. O diagnóstico administrativo e situacional como instrumento para o planejamento de açóes na estratégia saúde da família. Cogitare Enfermagem, Curitiba, v. 13, n. 3, p. 448-452, 2008. http:// dx.doi.org/10.5380/ce.v13i3.13044.

ROCHA, E. F.; KRETZER, M. R. Ações de reabilitação de pessoas com deficiência na estratégia da saúde da família da Fundação Zerbini e Secretaria Municipal de Saúde de São Paulo - Região Sudeste - Sapopemba/ Vila Prudente - período 2000/2006. Revista de Terapia Ocupacional da Universidade de São Paulo, São Paulo, v. 20, n. 1, p. 59-67, 2009. http://dx.doi.org/10.11606/ issn.2238-6149.v20i1p59-67.

SAMPAIO, R. F.; LUZ, M. T. Funcionalidade e incapacidade humana: explorando o escopo da classificação internacional da Organizaçáo Mundial da Saúde. Cadernos de Saúde Pública, Rio de Janeiro, v. 25, n. 3, p. 475-483, 2009. http://dx.doi.org/10.1590/S0102-311X2009000300002. PMid:19300836. 
SANTOS, W. R. Deficiência e BPC: o que muda na vida das pessoas atendidas. Ciência \& Saúde Coletiva, Rio de Janeiro, v. 16, n. SE 1, p. 787-796, 2011. http:// dx.doi.org/10.1590/S1413-81232011000700009. PMid:21503425.

SÃO PAULO. Conselho Municipal de Saúde. Secretaria Estadual de Saúde de São Paulo. Secretaria Municipal de Saúde de São Paulo. Secretaria Municipal da Pessoa com Deficiência e Mobilidade Reduzida. Rede de cuidados à pessoa com deficiência RRAS-06: documento orientador. São Paulo, 2013. Elaborado para orientação do Grupo Condutor Estadual da Rede de Cuidados à Pessoa com Deficiência.

SOUZA, C. C. B. X.; ROCHA, E. F. Portas de entrada ou portas fechadas? $\mathrm{O}$ acesso à reabilitação nas Unidades Básicas de Saúde da região sudeste do município de Sáo Paulo - período de 2000 a 2006. Revista de Terapia Ocupacional da Universidade de São Paulo, São Paulo, v. 21, n. 3, p. 230-239, 2010. http://dx.doi.org/10.11606/ issn.2238-6149.v21i3p230-239.

SOUZA, F. R.; PIMENTEL, A. M. Pessoas com deficiência: entre necessidades e atenção à saúde. Cader- nos de Terapia Ocupacional da UFSCar, São Carlos, v. 20, n. 2, p. 229-237, 2012. http://dx.doi.org/10.4322/ cto. 2012.024 .

SOUZA, J. M.; CARNEIRO, R. Universalismo e focalização na política de atenção à pessoa com deficiência. Saúde e Sociedade, São Paulo, v. 16, n. 3, p. 69-84, 2007. http://dx.doi.org/10.1590/S010412902007000300007.

STARFIELD, B. Atenção primária equilibrio entre necessidades de saúde, serviços e tecnologia. Brasília: UNESCO, 2002.

TABOADA-LUGO, N.; MINAYA-RAMOS, G. Caracterización clínica y etiológica de las diferentes discapacidades en el Estado Plurinacional de Bolivia, 20092010. Revista Peruana de Epidemiologia, Peru, v. 16, n. 3, p. 1-7, 2012. Disponível em: <http://rpe.epiredperu.net/rpe_ediciones/2012_V16_n03/6AO_Vol16_ No3_2012_discapacidad_Bolivia.pdf>. Acesso em: 20 jan. 2015.

\section{Contribuição dos Autores}

Marta Aoki e Sabrina de Mello Rodrigues contribuíram com a concepção do texto, a pesquisa e a organização das fontes, a análise de dados e a redação do texto. Fátima Corrêa Oliver contribuiu com a orientação de todo o trabalho, a análise dos resultados, a organização dos dados e das fontes, e a revisão final do texto. Todas as autoras aprovaram a versão final do artigo.

\section{Notas}

${ }^{1}$ Participaram como pesquisadores em campo estudantes bolsistas do curso de graduação em terapia ocupacional da Universidade de Sáo Paulo O estudo foi aprovado pelos comitês de ética em pesquisa em seres humanos da Faculdade de Medicina da Universidade de São Paulo (Processo n. . 078/11) e da Secretaria Municipal de Saúde de São Paulo (Processo n. ${ }^{\circ}$ 130/11). Os participantes foram informados e assinaram o Termo de Consentimento Livre e Esclarecido. 